Europhysics Letters

PREPRINT

\title{
Two-dimensional shear modulus of a Langmuir foam
}

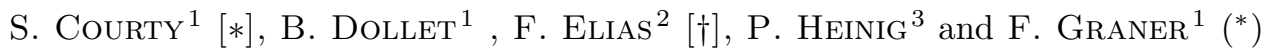 \\ 1 Laboratoire de Spectrométrie Physique (UMR 5588 CNRS - Université J. Fourier \\ Grenoble 1), BP 87, F-38402 Saint Martin d'Hères cedex, France \\ 2 Laboratoire des Milieux Désordonnés et Hétérogènes (UMR 7603 CNRS - Université \\ Paris 6), case 78, 4 place Jussieu, F-75252 Paris cedex 05, France \\ 3 Max Planck Institut of Colloids and Interfaces, Am Mühlenberg 1, D-14476 Golm, \\ Germany
}

PACS. 83.80.Iz - Emulsions and foams.

PACS. 68.18.-g - Langmuir films on liquids.

PACS. 62.20.Dc - Elasticity, elastic constants.

\begin{abstract}
We deform a two-dimensional (2D) foam, created in a Langmuir monolayer, by applying a mechanical perturbation, and simultaneously image it by Brewster angle microscopy. We determine the foam stress tensor (through a determination of the $2 \mathrm{D}$ gas-liquid line tension, $2.35 \pm 0.4 \mathrm{pJ} \cdot \mathrm{m}^{-1}$ ) and the statistical strain tensor, by analyzing the images of the deformed structure. We deduce the $2 \mathrm{D}$ shear modulus of the foam, $\mu=38 \pm 3 \mathrm{nN} \cdot \mathrm{m}^{-1}$. The foam effective rigidity is predicted to be $35 \pm 3 \mathrm{nN} \cdot \mathrm{m}^{-1}$, which agrees with the value $37.5 \pm 0.8 \mathrm{nN} \cdot \mathrm{m}^{-1}$ obtained in an independent mechanical measurement.
\end{abstract}

Introduction. - A liquid foam, made of polyhedral gas bubbles separated by thin liquid walls forming a connected network [1, is a mixture of two fluids. It has nevertheless a solidlike elasticity, characterised by a shear modulus $\mu$, proportional to the surface tension of the walls 22 3. In fact, shearing a foam modifies the total length of the walls, thus the foam energy. The value of $\mu$ can be determined in numerical simulations [4, 5, 6]; however, it is still an open problem to predict analytically its value for a real foam, which has a finite fluid fraction and an inherent disorder due to its distribution of bubble sizes.

Here, we compare two experimental measurements of $\mu$. First, by global mechanical measurements on the scale of the whole foam, described in terms of elasticity of continuous media. Second, and simultaneously, by detailed imaging of the diphasic foam structure, on the local level of a few bubbles: this suggests to use two-dimensional (2D) foams. In the literature, 2D soap froths have been sheared in Couette geometry, either as bubble rafts [7] 8, 9] or confined in Hele-Shaw cells between two parallel plates of glass [10.

We investigate the elasticity of a real 2D system: a "Langmuir foam" [11. A monomolecular layer of amphiphilic molecules deposited at the surface of water ("Langmuir monolayer") exhibits a first order transition between a 2D gas phase and a denser 2D liquid (also called

$\left(^{*}\right)$ Author for correspondence at graner@ujf-grenoble.fr. Fax: (+33) 476635495.

(c) EDP Sciences 
"liquid-expanded") phase. In the 2D gas-liquid coexistence region, the domains spontaneously arrange into a foam [11. Walls are stabilised by electrostatic dipolar interactions of the molecule themselves, without any added external surfactant 12, 13. Such Langmuir foams are approximately characterised by a line tension (see below), hence obey the same Plateau rules as other 2D liquid foams [14, 15], and display the same generic rheological behavior [16].

At the global level, we probe the foam effective rigidity $k_{\text {eff }}$ by measuring the force exerted on a rigid obstacle moving relatively to the foam [17. Independently, at the local level, we determine the foam stress tensor (through a determination of the 2D gas-liquid line tension) and the statistical strain tensor, by analyzing the bubble deformation 18; we deduce the 2D shear modulus of the foam 19. We then compare both measurements, in the frame of linear elasticity of continuous isotropic media [20].

Methods. - The 2D Langmuir foam is formed at $T=21^{\circ} \mathrm{C}$ in a home-made Langmuir teflon (PTFE) trough, of dimension $a \times b=8 \times 2 \mathrm{~cm}^{2}, 0.7 \mathrm{~cm}$ deep. Pentadecanoic acid $\left(\mathrm{C}_{14} \mathrm{H}_{29} \mathrm{CO}_{2} \mathrm{H}\right)$ is dissolved in chloroform at a concentration of $3 \cdot 10^{-4} \mathrm{~mol} \cdot \mathrm{l}^{-1}$. In order to prevent the dissociation of acid, the $\mathrm{pH}$ of the ultrapure water is set at $\mathrm{pH}=2$ by adding hydrochloric acid 14 15]. The air-water interface is cleaned by aspiration, then $\sim 10 \mu \mathrm{l}$ of the solution are spread onto the surface of water. After 10 minutes, the chloroform is evaporated, and a Langmuir foam of pentadecanoic acid forms. Using teflon compression barriers, we choose to adjust the surface fraction of $2 \mathrm{D}$ liquid to the limit at which walls are robust and clearly visible, which is about $23 \%$. With an average bubble size $\sim 100 \mu \mathrm{m}$, bubbles are large enough for image analysis, and there are enough bubbles to perform statistics.

The experimental set-up, inspired from ref. [21], is presented in ref. [17]. We determine the resistance exerted by the foam on an obstacle displaced relatively to the foam in the horizontal plane. The obstacle is the tip of a vertical rod, actually a denuded glass optical fiber (Thorlabs), which also acts as force sensor. Since the shear modulus of the Langmuir foam is low, we must here take a much softer fiber than in ref. 17. We chose a length $L=3.5 \pm 0.1 \mathrm{~cm}$, then attack the glass fiber with fluorhydric acid at $40 \%$ concentration until we reach its core, of diameter $2 r=6 \pm 1 \mu \mathrm{m}$.

The lower end of the fiber plunges vertically in the foam and immerses $10 \mu \mathrm{m}$ below the surface. The fiber is silanised (n-octadecyltrichlorosilane diluted at $2 \%$ in octane): the contact angle, measured with a camera attached to the side of the trough, is close to $90^{\circ}$, and the residual meniscus is small (white region on Fig. 1p, c).

The upper end of the fiber is held in a concentric chuck fixed on a horizontal translation stage coupled to a motor, allowing for a horizontal displacement $X_{\mathrm{imp}}$ : this applies a horizontal point-like deformation to the monolayer. The horizontal deflection $\zeta$ of the free end of the fiber is then measured by connecting the fiber to a laser diode, and collecting (in a photodetector placed under the trough) the beam transmitted at the free end of the fiber. We then deduce the horizontal resistance force $F$ exerted by the foam on the vertical fiber as $F=K \zeta$. Here, the fiber rigidity $K$ (calibrated by holding the fiber horizontally at one end, and measuring its deflection under its own weight) is $K=362 \pm 30 \mathrm{nN} \cdot \mathrm{m}^{-1}$, suitable for precise measurements in the $\mathrm{pN}$ range.

The foam effective rigidity $k_{\text {eff }}$, i.e. the ratio of the force $F$ to the horizontal displacement $X=X_{\mathrm{imp}}-\zeta$ of the fiber within the foam, is measured as $k_{\mathrm{eff}}=F / X=K \zeta / X$. It characterises the whole foam (it depends on its elastic moduli, as well as its size and geometry) under a given applied deformation, as long as it remains elastic. It is physically intuitive: $k_{\text {eff }}$ represents the rigidity one would feel by sticking a finger in the foam and moving it laterally.

The contrast and resolution of the Brewster angle microscope 23] have been optimised to image this foam; for details see ref. 25] pp. 54-60. Briefly, an incident He-Ne (Uniphase, 30 

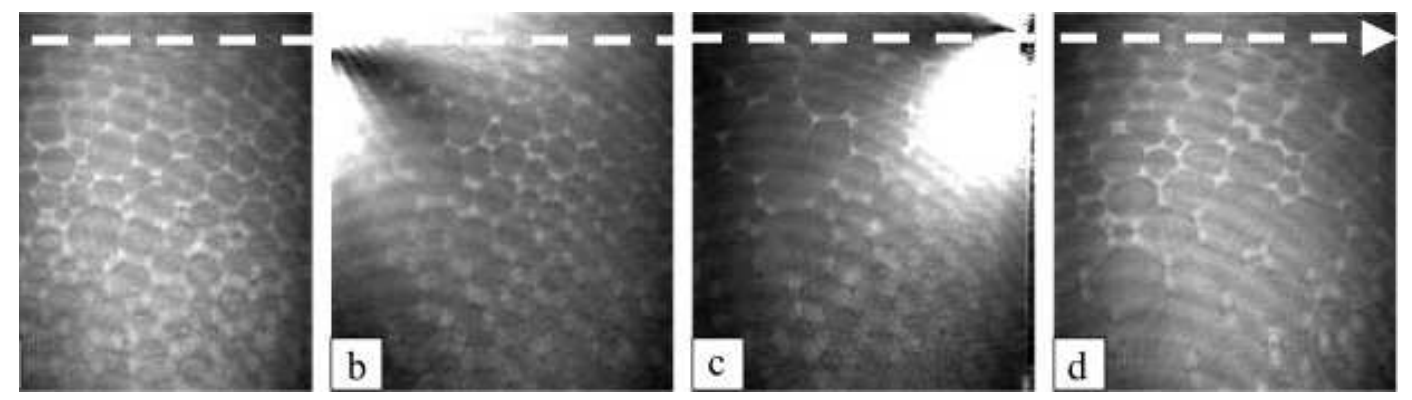

Fig. 1 - Four images of the movie 22: (a) $10.5 \mathrm{~s}$ before, (b) $0.7 \mathrm{~s}$ before, (c) $0.7 \mathrm{~s}$ after and (d) $28.4 \mathrm{~s}$ after the fiber passes through the middle of the field of view. The fiber moves from the left to the right (white arrow) at a constant applied velocity $\dot{X}_{\text {imp }}=620 \mu \mathrm{m} \cdot \mathrm{s}^{-1}$. The size of each image (here corrected by the projection factor at Brewster angle) is $768 \times 850 \mu \mathrm{m}^{2}$. The $2 \mathrm{D}$ gas phase of the monolayer, of low density, corresponds to a zero reflected intensity and appears black on the images, whereas the liquid phase, denser, appears brighter 23]. The average area is larger on the last images than on the first ones: this is due to the large-scale inhomogeneities (unavoidable with our preparation method) made apparent by the bulk flow, and not to the increase of area of each bubble separately (which contributes less than $6 \%$ during our experiment, and is likely due to a partial solubilization of amphiphilic molecules in water, rather than to actual coarsening [24]). We do not observe wall breakage (it appears only at 4 times higher velocity, or with a stiffer fiber), nor perturbation of the liquid-gas coexistence.

$\mathrm{mW}, 632.88 \mathrm{~nm}$ ) laser beam is tilted at an incident angle equal to the Brewster angle of the air-water interface $i_{\mathrm{B}} \approx 53^{\circ}$, passes a Glan Thomson polariser (Melles-Griot) in the plane of incidence, and a quarter wave plate (Melles-Griot). After reflexion on the surface of water, the beam enters a combination of two objectives (Zeiss) with small numerical aperture (NA $=0.1$ ), magnifying 5 and 10 times, respectively, and an analyser (Newport). The image of the surface forms on a CCD camera (sensitivity $10^{-3}$ lux) and is recorded simultaneously on video and on computer via a Scion Image frame grabber.

On each image (Fig. 1), we measure the contribution of the wall network to the stress tensor, as follows [26]. In a 2D foam, a wall represents two gas-liquid interfaces: thus the wall tension is $\tau=2 \lambda$, where $\lambda$ is the gas-liquid line tension (see below). The network contribution to the stress $\overline{\bar{\sigma}}$ could be measured from the image, by identifying the bubble walls which cross a given line of unit length and normal vector $\vec{v}$. The vectorial sum of their tensions [20], here $\vec{\tau}=2 \lambda \hat{e}$, where $\hat{e}$ is the unit vector tangent to the wall (its orientation is unimportant in what follows), would measure $\overline{\bar{\sigma}} \cdot \vec{v}$. As shown in ref. 18, we measure the stress tensor with better statistics [19] if we use its equivalent definition over the bulk of the image [27, which writes here (neglecting the curvature of walls [28]): $\overline{\bar{\sigma}}=S^{-1} \sum \vec{\tau} \otimes \vec{\ell}$, where $S$ is the area of the image, $\vec{\ell}=\ell \hat{e} ; \ell$ is the length of the wall; the sum is taken over all the walls present on the image; $\otimes$ is the tensor product: $\sigma_{i j}=S^{-1} \sum \tau_{i} \ell_{j}$ [18].

We thus need to determine the gas-liquid line tension $\lambda$ (Fig. 2a). We adapt the method of ref. 31, as follows. We record images in fluorescence microscopy, with 1\% NBD-HDA dye (all other conditions being unchanged). With a localised laser heating $(2 \mathrm{~K})$, we break one wall. The resorption of the broken wall's free extremity is driven by the wall tension

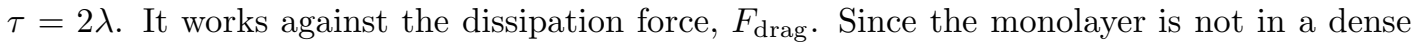
phase, the usual 3D viscosity $\eta_{3 \mathrm{D}}$ of the water subphase dominates the $2 \mathrm{D}$ one [32, 33, 34, 


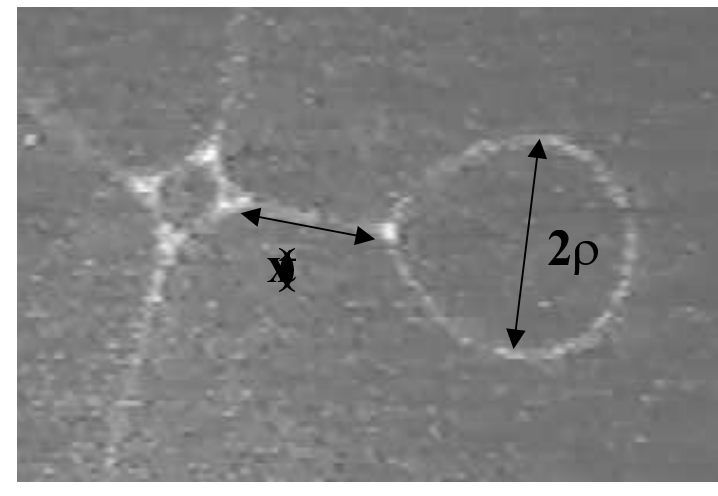

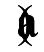

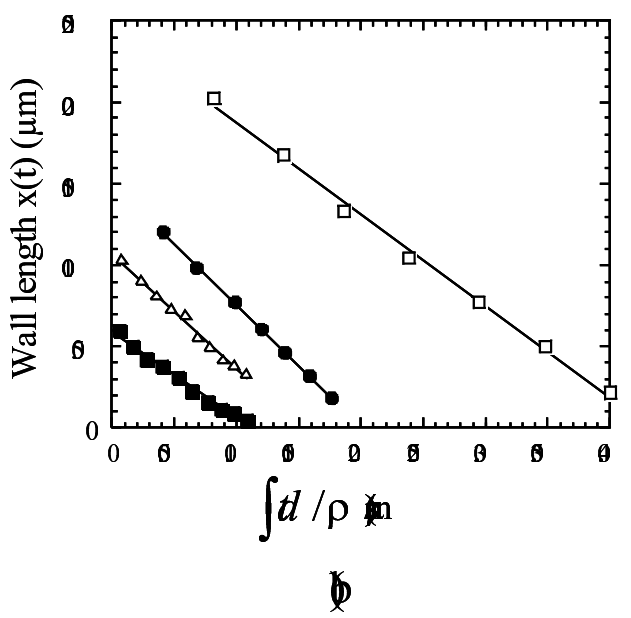

Fig. 2 - Measurement of the line tension $\lambda$. (a) Image $\left(320 \times 215 \mu \mathrm{m}^{2}\right)$ by fluorescence microscopy. By breaking a wall (on the right of the image, not shown), we obtain a bubble (radius $\rho$ ), attached to a single wall (length $x$ ), which retracts due to the line tension. (b) Plot of wall length $x(t)$ versus $\int_{0}^{t} d t / \rho(t)$ (see text) for four experiments with different initial length $x(0)$. We obtain straight lines, which proves that, at these length scales, the line tension is constant despite long-range dipolar interactions [12 13, 24] 30].

and: $F_{\text {drag }}=-\left(3 \pi^{2} / 4\right) \rho \eta_{3 \mathrm{D}} \dot{x}$. Here $\dot{x}$ is the rate of retraction of the length $x$ of the wall; $\rho$ is the radius of the object at the end of the wall. We chose the largest possible $\rho$ (to enhance sensitivity): here, a free bubble remains attached at the end of the wall (Fig. 2a). The prefactor $\left(3 \pi^{2} / 4\right) \approx 7.4$ is calculated at the limit of vanishing surface viscosity (zero Boussinesq number) for a deformable object (here: the bubble) [35], as opposed to a solid object which would lead to a prefactor of 8 [30, 34. We then deduce $\lambda$ from the balance of both forces. Since the bubble radius $\rho(t)$ can vary with time, we write: $x(t)-x(0)=-8 \lambda\left(3 \pi^{2} \eta_{3 \mathrm{D}}\right)^{-1} \int_{0}^{t} d t / \rho(t)$. From the slopes of Fig. (2), we measure $\lambda=2.35 \pm 0.4 \mathrm{pJ} \cdot \mathrm{m}^{-1}$.

To quantify the bubble anisotropy, we use the local texture tensor $\overline{\bar{M}}$ 18. It is a tensor constructed using all walls in a local region (here: the field of view) of the foam: $\overline{\bar{M}}=\langle\vec{\ell} \otimes \vec{\ell}\rangle$, i.e. $M_{i j}=\left\langle\ell_{i} \ell_{j}\right\rangle$, where $\vec{\ell}$ is the vector linking both ends of a wall, and \langle\rangle stands for an average over the walls [18. Its logarithm $\ln \overline{\bar{M}}$ has the same axes as $\overline{\bar{M}}$, and is real and symmetric 36. We use it to define the statistical strain tensor $\overline{\bar{U}}=\left(\ln \overline{\bar{M}}-\ln \overline{\bar{M}}_{0}\right) / 2$, where $\overline{\bar{M}}_{0}$ is the reference value of $\overline{\bar{M}}$ in the undeformed state of the foam. This tensor $\overline{\bar{U}}$ reduces to the usual definition of strain in the validity limits of classical elasticity [18].

We measure the two components $U_{x y}$ and $U_{x x}-U_{y y}$ : they are independent of $\overline{\bar{M}}_{0}$; in a weakly deformed material, they are roughly equal to $\left\langle\ell_{x}^{2}-\ell_{y}^{2}\right\rangle /\left\langle\ell_{x}^{2}+\ell_{y}^{2}\right\rangle$ and $\left\langle\ell_{x} \ell_{y}\right\rangle /\left\langle\ell_{x}^{2}+\ell_{y}^{2}\right\rangle$, respectively. We then compare them to the corresponding components of the stress tensor (Fig. [3a) [19]: the relation between the stress and strain tensors defines the shear modulus $\mu$ 20. In fact, in a 2D linear, homogeneous and isotropic medium, where the stress has no vertical diagonal component ("plane stress", $\sigma_{z z}=0$ ), the $2 \mathrm{D}$ Hooke law defines $\mu$, and the 

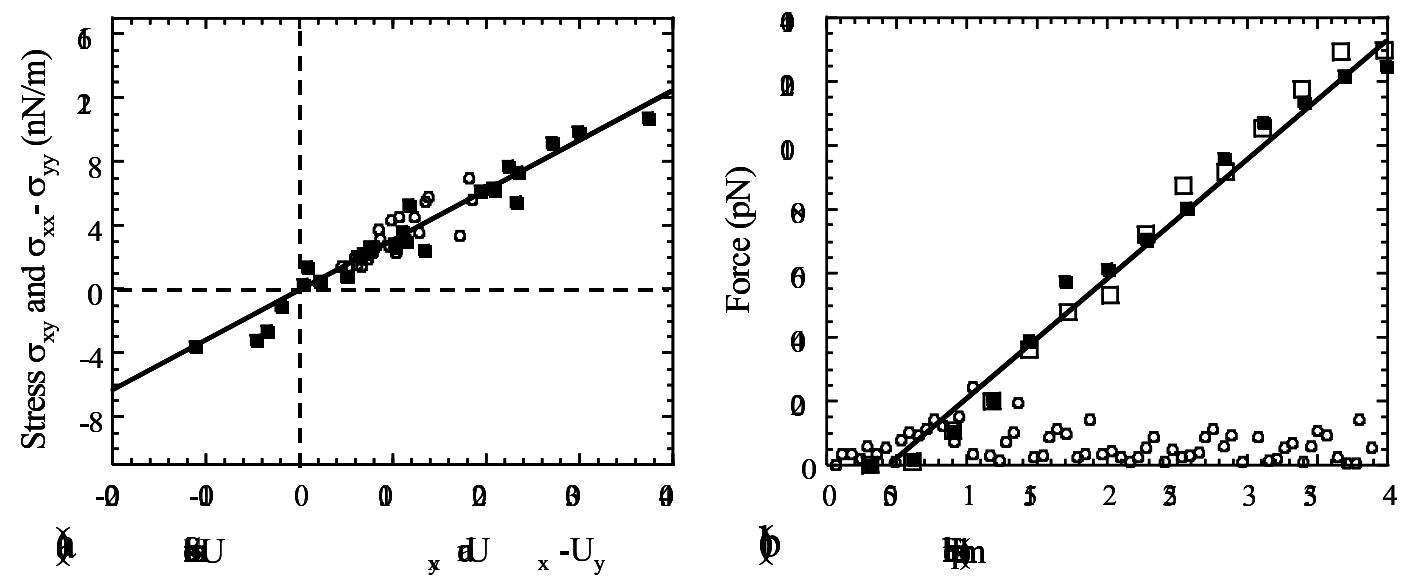

Fig. 3 - Two independent determinations of the Langmuir foam elasticity. (a) Measurement of the shear modulus $\mu$ based on analysis of 28 images. Each image provides two points, one for each component: closed squares: normal stress difference $\sigma_{x x}-\sigma_{y y}$ versus $U_{x x}-U_{y y}$; open circles: shear stress $\sigma_{x y}$ versus $U_{x y}$. Solid line: linear fit through all points, slope $76 \pm 6 \mathrm{nN} \cdot \mathrm{m}^{-1}$. (b) Mechanical measurement of the effective rigidity $k_{\text {eff }}$. The force $F=K \zeta$ exerted by the foam on the fiber is plotted versus the displacement $X=X_{\mathrm{imp}}-\zeta$ of the fiber free end. Closed squares: the fiber is displaced in the direction $X_{\mathrm{imp}}>0$ at a constant velocity of $620 \mu \mathrm{m} \cdot \mathrm{s}^{-1}$. Open squares: it returns back to its initial position at the same velocity. Solid line: linear fit through all points, slope $37.5 \pm 0.8 \mathrm{nN} \cdot \mathrm{m}^{-1}$. Open circles: control experiment, at the surface of pure water.

2D Poisson ratio $\nu(-1 \leq \nu \leq 1)$, through [17]:

$$
\sigma_{x x}+\sigma_{y y}=2 \mu \frac{1+\nu}{1-\nu}\left(U_{x x}+U_{y y}\right), \quad \sigma_{x x}-\sigma_{y y}=2 \mu\left(U_{x x}-U_{y y}\right), \quad \sigma_{x y}=2 \mu U_{x y} .
$$

Results. - Initially (Fig. 目), the bubbles are relaxed and the foam is isotropic. The fiber approaches the field of view (Fig. 10) and compresses the bubbles. Behind the fiber (Fig. 11), the bubbles are stretched. After relaxation (Fig. 11) the foam becomes again isotropic. Fig. (3i) shows a plot of the elastic normal stress difference $\sigma_{x x}-\sigma_{y y}$ versus $U_{x x}-U_{y y}$, and of the shear stress $\sigma_{x y}$ versus $U_{x y}$. All the data collapse on the same straight line; its slope measures $2 \mu$ (eq. 10):

$$
\mu=38 \pm 3 \mathrm{nN} \cdot \mathrm{m}^{-1} .
$$

This value can be compared to the theoretical computation for a $2 \mathrm{D}$ foam with a regular, dry honeycomb structure [27, 37, 38, of bubble area $A: \mu_{\mathrm{h}}=0.465 \times(2 \lambda) A^{-1 / 2}$. For our system, $2 \lambda=4.7 \mathrm{pJ} \cdot \mathrm{m}^{-1}$, the average bubble area $\bar{A}^{-1 / 2}=1.7 \cdot 10^{4} \mathrm{~m}^{-1}$, and this theoretical expression would give a prediction, $\mu=37.2 \mathrm{nN} \cdot \mathrm{m}^{-1}$, similar (within errors) to our measurement from image analysis. A previous experimental measurement on a dry (fluid fraction $<3 \%$ ) 2 D soap froth found $\mu$ higher than for the honeycomb of $20 \%$, and suggested to interpret it as an effect of disorder in wall lengths [19. Here, we cannot discuss such effect, since it would be counterbalanced by the effects of the fluid fraction (our 2D wet foam is expected to have a lower $\mu$ than the theoretical dry one [1], similarly to what happens in 3D [6, 39]).

This local measurement of the shear modulus leads to a testable prediction: the value of the global foam rigidity, experienced by the free end of the fiber. The foam rigidity $k_{\text {eff }}$ is a 
EUROPHYSICS LETTERS

function of $\mu$ and $\nu$; due to the logarithmic range of $2 \mathrm{D}$ elasticity [17, the rigidity also depends on the set-up geometry and the boundary conditions at the edges of the trough, which we do not know. We use for simplicity the calculations for non-slip boundary conditions [17. Here the fiber radius is $r=3 \mu \mathrm{m}$, the trough width and length are $a=b=2 \mathrm{~cm}$, and we obtain:

$$
k_{\mathrm{eff}}=\frac{\mu}{1.085-0.362 \nu} .
$$

The foam Poisson ratio $\nu$, which is between -1 and 1 , is not measurable in our experiment. Since it is probably determined mostly by the network of bubble walls (in the liquid-gas coexistence region, the pressure inside the bubbles is constant [14, hence does not contribute to the foam compressibility), it is likely to be much smaller than unity 40: this is compatible with the visual impression 22. If we neglected it for simplicity, eq. (2) would yield, using our measured value $\mu=38 \pm 3 \mathrm{nN} \cdot \mathrm{m}^{-1}$ :

$$
k_{\text {eff }}(\text { image })=35 \pm 3 \mathrm{nN} \cdot \mathrm{m}^{-1} .
$$

This value predicted under all the above assumptions can now be compared to the direct mechanical measurement of $k_{\text {eff }}$, shown on Fig. (3b). The subphase contribution to the force is negligible, as shown when performing the same experiment on the surface of pure water. During the deformation (Fig. 1), the foam apparently remains in its elastic regime. In fact, first, the force-displacement plot is affine over a displacement of $3 \mathrm{~mm}$, despite the (yet unexplained) initial time-lag visible on Fig. (3b). Second, as is visible on the movie [22], no bubble rearrangement and no wall breakage is observed. Third, even along the path of the fiber, the foam is intact: when the fiber is displaced backwards, the force-displacement plot is reversible. We find that its slope $k_{\text {eff }}$ agrees (within errors) with the above prediction:

$$
k_{\text {eff }}(\text { force })=37.5 \pm 0.8 \mathrm{nN} \cdot \mathrm{m}^{-1} .
$$

Perspectives. - This result illustrates how adapted is the statistical strain tensor to describe locally the elastic properties of disordered media. Beside foams, we intend to adapt

it to other amorphous materials, such as glasses and polymer networks, to investigate how the microscopic disorder of the structure affects the material's elasticity tensor.

We gratefully acknowledge the help and explanations of T. Fischer for the measurement of the line tension. We thank S. Akamatsu, M. Aubouy, P. Ballet, S. Hénon, K. Kassner and C. Quilliet for useful discussions. This work was partially supported by CNRS ATIP 0693.

\section{REFERENCES}

[*] Present address : Cavendish Laboratory, Madingley Road, Cambridge, CB3 0HE, UK.

[†] Affiliated to Fédération de Recherche FR2438 "Matière et Systèmes Complexes".

[1] Weaire D. and Hutzler S., Physics of Foams (Oxford Univ. Press, Oxford) 1999.

[2] Höhler R., Cohen-Addad S. and Hoballah H., Phys. Rev. Lett., 79 (1997) 1154.

[3] Cohen-Addad S., Hoballah H. and Höhler R., Phys. Rev. E, 57 (1998) 6897.

[4] Weaire D. and Fortes M. A., Advances in Physics, 43 (1994) 685.

[5] Kawasaki K., Okusono T. and Nagai T., J. Mech. Behav. Mat., 4 (1992) 51.

[6] Durian D. J., Phys. Rev. E, 55 (1997) 1739. 
[7] Lauridsen J., Twardos M. and Dennin M., Phys. Rev. Lett., 89 (2002) 098303.

[8] Abd el Kader A. and Earnshaw J. J., Phys. Rev. Lett., 82 (1998) 2610.

[9] Pratt E. and Dennin M., Phys. Rev. E, 67 (2003) 051402.

[10] Debrégeas G., Tabuteau H. and Di Meglio J.-M., Phys. Rev. Lett., 87 (2001) 17-8305.

[11] Lösche M., Sackmann E., and Möhwald H., Ber. Buns.-Ges. Phys. Chem., 87 (1983) 848

[12] Mann E. K. and Primak S. V., Phys. Rev. Lett., 83 (1999) 5397.

[13] Rivière S., Hénon S., Meunier J., Albrecht G., Boissonnade M. M., and Baszkin A., Phys. Rev. Lett., 75 (1995) 2506.

[14] Stine K. J., Rauseo S. A., Moore B. G., Wise J. A. and Knobler C. M., Phys. Rev. A, 41 (1990) 6884.

[15] Berge B., Simon A. J. and Libchaber A., Phys. Rev. A, 41 (1990) 6893.

[16] Dennin M. and Knobler C. M., Phys. Rev. Lett., 78 (1997) 2485.

[17] Courty S., Dollet B., Kassner K., Renault A. and Graner F., Eur. Phys. J. E, 11 (2003) 53.

[18] Aubouy M., Jiang Y., Glazier J. A. and Graner F., Granular Matt., 5 (2003) 67.

[19] Asipauskas M., Aubouy M., Glazier J. A., Graner F. and Jiang Y., Granular Matt., 5 (2003) 71.

[20] Landau L. D. and Lifschitz E. M., Theory of Elasticity (Reed, Oxford, $3^{\text {rd }}$ ed.) 1986.

[21] Barentin C., Ybert C., di Meglio J.-M. and Joanny J.-F., J. Fluid Mech., 397 (1999) 331.

[22] Movie available at http://www-lsp.ujf-grenoble.fr/link/mousses-films.htm

[23] HÉnon S. and Meunier J., Rev. Sci. Instrum., 62 (1991) 936.

[24] Heinig P. and Fischer T., to appear in J. Phys. Chem.

[25] Courty S., PhD thesis, Univ. Grenoble, France, 2001, unpublished. Available as .pdf file (16 Mo) at http://www-lsp.ujf-grenoble.fr/link/courty.htm

[26] Reinelt D. A. and Kraynik A., J. Rheology, 44 (2000) 453.

[27] Kraynik A. M., Annu. Rev. Fluid. Mech., 20 (1988) 325.

[28] In a quasistatic 2D foam, the laplacian of the pressure is zero [29], the bubble pressure (hence the wall curvature) induces only a negligible correction with respect to straight walls 19.

[29] Graner F., Jiang Y., Janiaud E., and Flament C., Phys. Rev. E, 63 (2001) 011402/1.

[30] Wurlitzer S., Steffen P., Wurlitzer M., Khattari Z., and Fischer T., J. Chem. Phys., 113 (2000) 3822.

[31] Wurlitzer S., Steffen P., and Fischer T., J. Chem. Phys., 112 (2000) 5915.

[32] Klingler J. and McConnell H., J. Phys. Chem, 97 (1993) 6096.

[33] Steffen P., Heinig P., Wurlitzer S., Khattari Z. and Fischer T.M., J. Chem. Phys., 115 (2001) 994.

[34] Wurlitzer S., Schmiedel H. and Fischer T.M., Langmuir , 18 (2002) 4393.

[35] DE Koker, R.E., PhD thesis, Stanford Univ., USA, 1996, unpublished, eq. 5.26; quoted in eq. (12) of ref. 34.

[36] By definition, the tensor $\ln \overline{\bar{M}}$ is calculated by rotating $\overline{\bar{M}}$ to a basis where it is diagonal, taking the logarithm of its eigenvalues, then rotating it back into the initial basis.

[37] Princen H. M., J. Coll. Int. Sci., 91 (1983) 160.

[38] Morse D. C. and Witten T. A., Europhys. Lett., 22 (1993) 549.

[39] Mason T.G., Bibette J. and Weitz D.A., Phys. Rev. Lett., 75 (1995) 2051.

[40] Boal D., Seifert U., and Shillcock J., Phys. Rev. E, 48 (1993) 4274. 